\title{
USING UNIVARIATE BÉZIER DISTRIBUTIONS TO MODEL SIMULATION INPUT PROCESSES
}

\author{
Mary Ann Flanigan Wagner \\ SysTech Software Solutions \\ P.O. Box 413 \\ Annandale, VA 22003, U.S.A.
}

\begin{abstract}
In this paper we describe a graphical, interactive technique for modeling univariate simulation input processes with a distribution family based on Bézier curves. This family has an open-ended parameterization and is capable of accurately representing an unlimited variety of distributional shapes. Our input-modeling technique is implemented in a self-contained, Windows-based software system called PRIME-PRobabilistic Input Modeling Environment. Several examples illustrate the application of this technique.
\end{abstract}

\section{INTRODUCTION}

One of the main problems in the design and construction of large-scale stochastic simulation experiments is the selection of valid input models-that is, probability distributions that accurately mimic the behavior of the stochastic input processes driving the system. Often the following difficulties arise when using standard distribution families for simulation input modeling: (a) many real-world input processes exhibit probabilistic behavior that cannot be represented faithfully with standard stochastic models; (b) there are a limited number of parameters available to control the shape of the fitted distributions; and (c) the parameters of these distributions are often difficult to estimate from either sample data or subjective information (expert opinion). Moreover, the user has no guarantee of conclusively identifying an appropriate distribution family using conventional graphical and statistical goodness-of-fit tests; and the user generally lacks a unique, well-formulated criterion by which to estimate the parameters of any given distribution family. The problem of modeling simulation input processes is further complicated if sample data are not available. In this case, the selection of a distribution is based arbitrarily on whatever informa-

\author{
James R. Wilson
}

Department of Industrial Engineering

North Carolina State University

Raleigh, NC 27695-7906, U.S.A.

tion can be elicited from knowledgeable individuals.

In this paper, we present a flexible, interactive, graphical methodology for modeling a broad range of input processes that arise in large-scale simulation studies. We implemented this methodology in a self-contained, Microsoft-Windows-based software system called PRIME-PRobabilistic Input Modeling Environment. PRIME integrates graphical and statistical concepts to form a tool that helps an analyst select and visualize an appropriate representation of a random input process using interactive-subjective, data-driven, and visually-based techniques. We have exploited the properties of Bézier curves to develop a flexible univariate distribution family that has an open-ended parameterization capable of accurately representing an unlimited number of distributional shapes.

The remainder of this paper is organized into four sections. In Section 2 we review the current techniques used for modeling stochastic simulation input processes. In Section 3 we introduce a methodology for input modeling with univariate Bézier distributions, and in Section 4 we describe the implementation of this methodology in PRIME. In Section 5 we present some examples to illustrate the application of this methodology. Finally, in Section 6 we summarize the main contributions of this work. This paper is based on Flanigan (1993).

\section{CONVENTIONAL TECHNIQUES FOR SIMULATION INPUT MODELING}

\subsection{Subjective Distribution Estimation}

Subjective estimation of a distribution is necessary when the process to be modeled lacks data. The data may be nonexistent, as is the case when a new process is being introduced, or data may be difficult to obtain due to cost or time constraints. If data are not available, then the selection of a distribution and estimation of its parameters must be based on other 
characteristics of the process.

There are several software packages that can be used to display and manipulate probability distributions in the absence of data. The INSIGHT simulation environment (Roberts 1983) displays standard distributions given user-specified parameters; IMSL (1987) can be used to plot standard distributions; and XCELL+ (Conway et al. 1987) displays the generalized Lambda distribution. All three software packages require the user to enter the parameters of the distribution directly, which presupposes detailed knowledge of the input process being modeled. Not one of these packages provides a means to edit the displayed distribution - each program must be restarted if a different parameter set is desired.

VISIFIT (DeBrota et al. 1989) and VIBES (AbouRizk, Halpin, and Wilson 1991) are designed for subjective estimation of bounded Johnson $\left(S_{B}\right)$ distributions and generalized beta distributions respectively. Both programs enable the user to edit graphically the shape of the density, thus altering the corresponding distribution parameters, until the fitted density is visually and subjectively acceptable. VISIFIT and VIBES have the following limitations. (a) Both programs are specifically tailored to distribution families that are limited to four parameters. (b) Both programs require complicated numerical methods for parameter estimation. (c) The user must make decisions about the shape of the distribution largely from subjective information-that is, VISIFIT and VIBES have only limited facilities for superimposing empirical distributions onto the viewing display.

\subsection{Data-Driven Distribution Estimation}

If data are available, a modeler is confronted with the problem of selecting a distribution that closely approximates both the underlying distribution and the sample data set. A simple distribution-fitting method is to use the empirical distribution corresponding to the sample data. The problem with using an empirical distribution is that its mass points constitute the entire support of the fitted distribution, and values that are not contained in the data set cannot be realized (Law and Kelton 1991).

Adding exponential tails to an empirical distribution is a method for extending the range of realizable values of the fitted distribution (Bratley, Fox and Schrage 1987). This method overcomes some of the deficiencies of the empirical distribution in modeling the tails of the underlying distribution, but it still requires a lot of information to construct an input model; and the final fitted distribution may not ad- equately represent the smoothness properties of underlying distribution.

UniFit II (Vincent and Law 1992) is a software package that is used to fit data to one of the standard probability distributions plus the more flexible Pearson type V and VI families, the Inverse Gaussian distribution, the extreme value distributions, and the logistic distribution. The program will select the "best" distribution to represent the data using a (proprietary) set of comparison and fitting schemes. The only method to tailor, or fine-tune, the chosen distribution is to alter the data set or to reset the bounds of the fitted distribution.

Swain, Venkatraman and Wilson (1988) developed FITTR1, a program that attempts to fit data to the entire Johnson system of distributions-namely the $S_{B}$ (bounded), $S_{U}$ (unbounded), $S_{L}$ (lognormal) and $S_{N}$ (normal) distribution families. FITTR1 incorporates an extensive battery of fitting algorithms to estimate the parameters of the best-fitting Johnson distribution for a given data set. However, because each Johnson distribution has at most four parameters, FITTR1 is not guaranteed to obtain a globally good fit to an arbitrary data set. Moreover, the parameter-estimation schemes in FITTR1 are computationally intensive, there are no graphical display capabilities, and methods to edit the fitted distribution are ad hoc.

Virtually all of the conventional methods for simulation input modeling have limited flexibility because they are based on distribution families with a fixed number of parameters. Hora (1983) and Avramidis and Wilson $(1989,1993)$ have introduced methods to extend the parameterization of distribution families. Unfortunately neither of these methods is guaranteed to yield a significantly improved fit; and Avramidis and Wilson (1993) show how Hora's method can actually yield a fit that is statistically inferior or even mathematically illegitimate. Moreover, the additional parameters introduced by these methods have no direct, intuitive interpretation in terms of the underlying process that is being modeled.

In view of the limitations of conventional techniques for simulation input modeling, there is a need for a parametrically extensible distribution family with the following properties: (a) its parameters are meaningful to users and easily estimated from data, and (b) its density and distribution function can be readily edited graphically. These considerations motivated our examination of Bézier distributions. 


\section{BÉZIER DISTRIBUTIONS}

\subsection{Definition of Bézier Curves}

In computer graphics, a Bézier curve is used to approximate a smooth univariate function on a bounded interval by forcing the Bézier curve to approximate a given set of control points $\left\{\boldsymbol{p}_{i} \equiv\left(x_{i}, z_{i}\right): i=\right.$ $0,1, \ldots, n\}$. Formally, a Bézier curve of degree $n$ with control points $\left\{\boldsymbol{p}_{0}, \boldsymbol{p}_{1}, \ldots, \boldsymbol{p}_{n}\right\}$ is given parametrically by

$$
\begin{aligned}
\boldsymbol{P}(t) & =\left[P_{x}(t ; n, \boldsymbol{x}), P_{z}(t ; n, \boldsymbol{z})\right] \\
& =\sum_{i=0}^{n} B_{n, i}(t) \boldsymbol{p}_{i} \text { for } t \in[0,1]
\end{aligned}
$$

where $\boldsymbol{x} \equiv\left(x_{0}, x_{1}, \ldots, x_{n}\right)$ and $\boldsymbol{z} \equiv\left(z_{0}, z_{1}, \ldots, z_{n}\right)$, and where for $i=0,1, \ldots, n$, the blending function $B_{n, i}(t)$ is the well-known Bernstein polynomial

$$
B_{n, i}(t) \equiv \frac{n !}{i !(n-i) !} t^{i}(1-t)^{n-i} \text { for } t \in[0,1] \text {. }
$$

Bézier curves have two characteristics that are particularly important for graphically-based approximation of functions (Farin 1990):

1. A Bézier curve interpolates exactly its first and last control points; this means that the curve will pass through these control points.

2. A Bézier curve is edited under global control; this means that a change in one control point affects the shape of the entire curve.

Although Bézier curves are edited under global control, the effect of the $i$ th control point $\boldsymbol{p}_{i}$ on the shape of the curve is greatest when $t=i / n$. As $t$ increases from 0 to 1 , the effect of the initial control point $\boldsymbol{p}_{0}$ on the current curve coordinates $\boldsymbol{P}(t)$ decreases; and the effect of the final control point $\boldsymbol{p}_{n}$ on $\boldsymbol{P}(t)$ increases. In this sense, the control points act like "magnets", where the "magnetic attraction" of control point $\boldsymbol{p}_{i}$ is strongest when the independent variable $t=i / n$ so that the corresponding point $\boldsymbol{P}(t)$ is in the vicinity of $\boldsymbol{p}_{i}$. Bézier curves are used extensively in computer graphics because of their ease of implementation, intuitive construction, and numerical stability.

\subsection{The Bézier Distribution Function}

If $X$ is a continuous random variable with bounded support $\left[x_{*}, x^{*}\right]$, unknown CDF $F_{X}(\cdot)$, and unknown PDF $f_{X}(\cdot)$, then we can approximate $F_{X}(\cdot)$ with an appropriate Bézier curve of the form (1), where the control points $\boldsymbol{p}_{0}, \boldsymbol{p}_{1}, \ldots, \boldsymbol{p}_{n}$ have been arranged so as to ensure the basic requirements of a CDF: (a) $F_{X}(x)$ is monotonically nondecreasing in $x$; (b) $F_{X}\left(x_{*}\right)=0$; and (c) $F_{X}\left(x^{*}\right)=1$. By utilizing the Bézier property that control points $\boldsymbol{p}_{0}$ and $\boldsymbol{p}_{n}$ are exactly interpolated, we can ensure $F_{X}\left(x_{*}\right)=0$ if we take $\boldsymbol{p}_{0} \equiv\left(x_{*}, 0.0\right)$, and $F_{X}\left(x^{*}\right)=1$ if we take $\boldsymbol{p}_{n} \equiv\left(x^{*}, 1.0\right)$.

\subsection{The Bézier Density Function}

If $X$ is a continuous random variable as defined in $\S 3.2$, then we can show that the corresponding density $f_{X}(\cdot)$ is given parametrically by

$$
\boldsymbol{P}^{*}(t)=\left[P_{x}^{*}(t ; n, \boldsymbol{x}), P_{z}^{*}(t ; n, \boldsymbol{x}, \boldsymbol{z})\right],
$$

where

$$
P_{x}^{*}(t ; n, \boldsymbol{x})=P_{x}(t ; n, \boldsymbol{x}),
$$

and

$$
\begin{aligned}
P_{z}^{*}(t ; n, \boldsymbol{x}, \boldsymbol{z}) & =\frac{P_{z}(t ; n-1, \Delta \boldsymbol{z})}{P_{x}(t ; n-1, \Delta \boldsymbol{x})} \\
& =\frac{\sum_{i=0}^{n-1} B_{n-1, i}(t) \Delta z_{i}}{\sum_{i=0}^{n-1} B_{n-1, i}(t) \Delta x_{i}}
\end{aligned}
$$

for all $t \in[0,1]$. In equation (5), we take $\Delta \boldsymbol{x}$ $\equiv\left(\Delta x_{0}, \ldots, \Delta x_{n-1}\right)$ and $\boldsymbol{\Delta} \boldsymbol{z} \equiv\left(\Delta z_{0}, \ldots, \Delta z_{n-1}\right)$, where $\Delta x_{i} \equiv x_{i+1}-x_{i}$ and $\Delta z_{i} \equiv z_{i+1}-z_{i}(i=$ $0,1, \ldots, n)$ represent the corresponding first differences of the $x$ - and $z$-coordinates of the original control points $\left\{\boldsymbol{p}_{0}, \boldsymbol{p}_{1}, \ldots, \boldsymbol{p}_{n}\right\}$ in the parametric representation (1) of the distribution function.

\subsection{Moments of the Bézier Distribution}

The expected value of a Bézier variate $X$ is given by

$$
E[X]=\frac{1}{2} \sum_{l=0}^{n}\left(\begin{array}{l}
n \\
l
\end{array}\right) x_{l} \sum_{i=0}^{n-1} \frac{\left(\begin{array}{c}
n-1 \\
i
\end{array}\right)}{\left(\begin{array}{c}
2 n-1 \\
l+i
\end{array}\right)} \Delta z_{i} .
$$

Closed-form expressions analogous to (6) can be given for the higher-order noncentral moments of a Bézier variate (Flanigan 1993), but these expressions are cumbersome to evaluate. An efficient alternative scheme to determine the moments of the distribution can be based on the following result

$$
E\left[X^{k}\right]=\int_{0}^{1} k\left[P_{x}(t)\right]^{k-1}\left[1-P_{z}(t)\right]\left|P_{x}^{\prime}(t)\right| d t .
$$

Equation (7) is valid when $X$ is a nonnegative random variable. If $X$ has a (finite) negative lower bound $x_{*}$, then equation $(7)$ can be applied to calculate the noncentral moments of the nonnegative random variable $\zeta_{*} \equiv X-x_{*}$. It follows immediately that 
$E[X]=E\left[\zeta_{*}\right]+x_{*} ;$ and the remaining central moments of $X$ coincide with the corresponding central moments of $\zeta_{*}$. Thus the standard deviation, the skewness, and the kurtosis of $X$ are the same as for $\zeta_{*}$.

\subsection{Generating Bézier Variates by Inversion}

A Bézier random variable with density (3) can be generated by inversion as follows. Given a random number $U \sim$ Uniform[0,1], we perform the following steps: (a) find $t_{U} \in[0,1]$ such that

$$
P_{z}\left(t_{U} ; n, z\right)=\sum_{i=0}^{n} B_{n, i}\left(t_{U}\right) z_{i}=U ;
$$

and (b) deliver the variate

$$
X=P_{x}\left(t_{U} ; n, \boldsymbol{x}\right)=\sum_{i=0}^{n} B_{n, i}\left(t_{U}\right) x_{i} .
$$

The solution to (8) can be found by any root-finding algorithm such as Müller's, Newton's, or the Bisection method (Conte and de Boor 1980).

\section{MODELING BÉZIER DISTRIBUTIONS USING PRIME}

PrIME, PRobabilistic Input Modeling Environment, is a graphical interactive software system that incorporates the methodology defined in $\S 3$ to help an analyst estimate the univariate input processes that arise in large-scale simulation studies. PrIME is designed for IBM-compatible microcomputers equipped with a math coprocessor and a pointing device such as a mouse. It is written entirely in the $\mathrm{C}$ programming language, and it has been developed to run under $\mathrm{Mi}$ crosoft Windows (Microsoft Corporation 1990) version 3.0 or later. PRIME is designed to be easy and intuitive to use. The construction of a CDF is performed through the actions of the mouse, and several options are conveniently available through menu selections. Control points are represented as small black squares, and each control point is given a unique label corresponding to its index $i$ in equation (1). Figure 1 shows a typical session in PRIME, where the CDF window is displayed.

In the absence of data, Prime can be used to describe an input process conceptualized from subjective information or expertise. The representation of the conceptualized distribution is achieved by adding, deleting, and moving the control points via the mouse. As mentioned in $§ 3.1$, each control point acts like a "magnet" pulling the curve in the

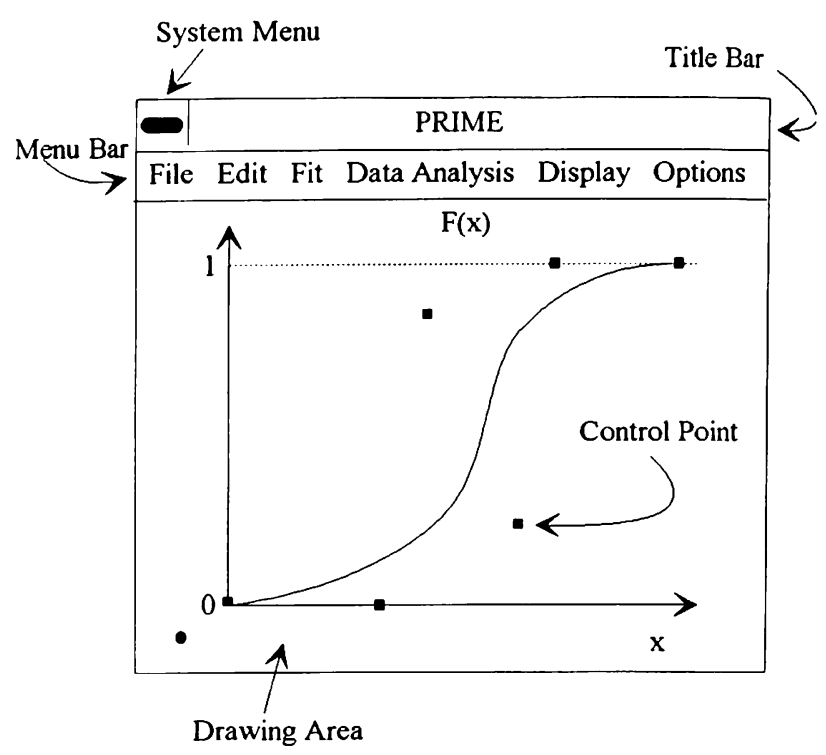

Figure 1: A Prime Window

direction of the control point, where the blending functions, described by the Bernstein polynomials, govern the strength of the "magnetic attraction" of each control point. The movement of a control point causes the displayed CDF to be updated (nearly) instantaneously. The corresponding PDF and the first four moments are (nearly) simultaneously updated in adjacent windows so that the user gets immediate feedback on the effects of his movements of the control point. Figures 2 and 3 give some indication of the wide variety of distributional shapes that can be achieved with this approach to subjective distribution estimation. The figures make it clear that the flexibility of this approach greatly exceeds that of subjective estimation techniques based on distribution families with a fixed number of parameters (DeBrota et al. 1989).

\subsection{Data-Driven Estimation of Bézier Distri- butions}

Classical fitting algorithms have been employed to fit Bézier distributions to sample data sets. Suppose that a random sample $\left\{X_{j}: j=1,2, \ldots, m\right\}$ has been taken from $F_{X}(\cdot)$ and that $F_{m}(x) \equiv$ (number of $X_{j}$ 's $\leq x) / m$ denotes the corresponding empirical distribution function. If we define an appropriate distance function $d\left(F_{m}(\cdot), \hat{F}_{\boldsymbol{X}}(\cdot ; n, \boldsymbol{x}, \boldsymbol{z})\right)$ between the empirical $\operatorname{CDF} F_{m}(\cdot)$ and the fitted $\operatorname{CDF} \hat{F}_{X}(\cdot ; n, \boldsymbol{x}, \boldsymbol{z})$ given parametrically by (1), then a natural approach to data-based distribution fitting is to use a suitable 


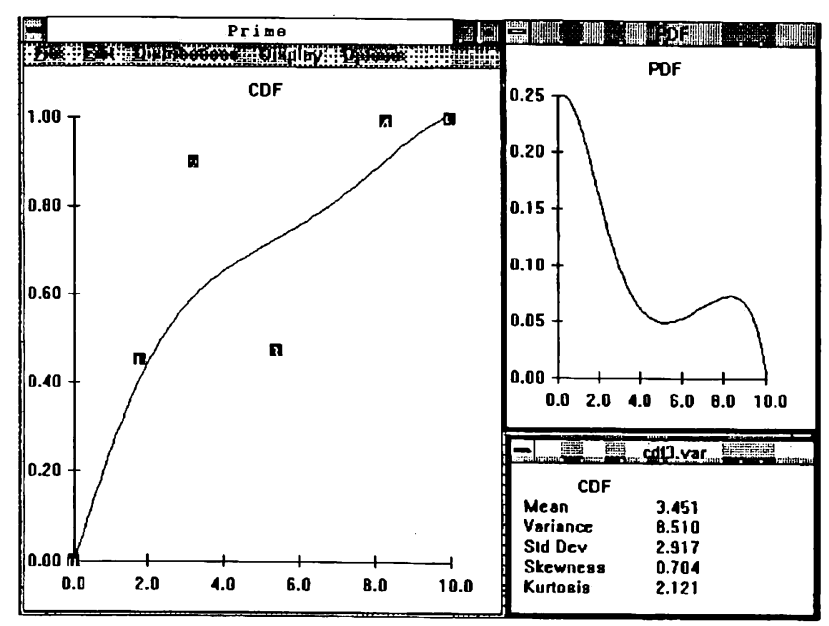

Figure 2: A Bimodal Distribution

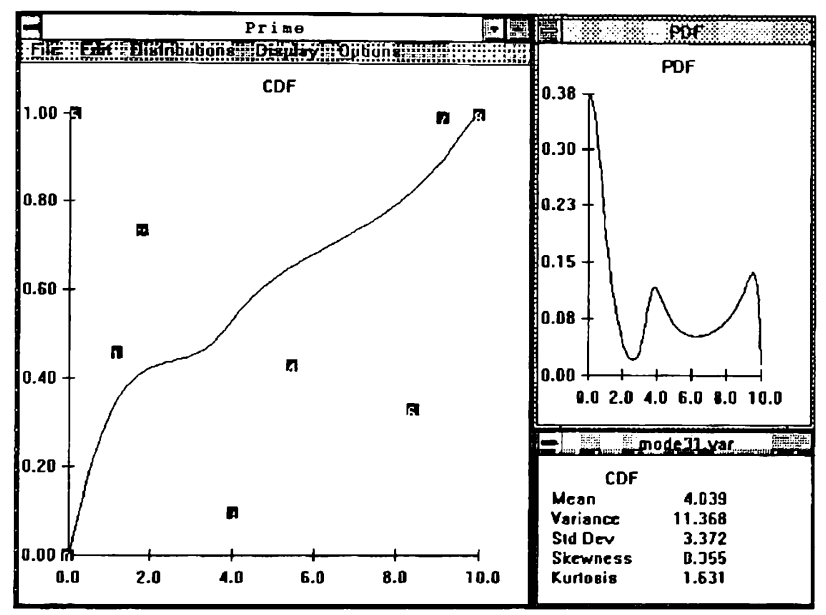

Figure 3: A Trimodal Distribution

optimization procedure to solve the problem

$$
\left.\begin{array}{cl}
\min _{\boldsymbol{x}, \boldsymbol{z}} & d\left(F_{m}(\cdot), \hat{F}_{X}(\cdot ; n, \boldsymbol{x}, \boldsymbol{z})\right) \\
\text { s. t. } & P_{z}^{*}(t ; n, \boldsymbol{x}, \boldsymbol{z}) \geq 0 \text { for } t \in[0,1] \\
& z_{0}=0, \quad z_{n}=1 \\
& x_{0} \leq X_{(1)}, \quad x_{n} \geq X_{(m)}
\end{array}\right\}
$$

where $X_{(1)} \leq X_{(2)} \leq \cdots \leq X_{(m)}$ are the order statistics for the sample $\left\{X_{j}\right\}$.

The classical fitting algorithms that have been incorporated into PRIME include: least-squares estimation, minimum- $L_{1}$-norm estimation, minimum$L_{\infty}$-norm estimation, maximum likelihood estimation, moment matching, and percentile matching. See Flanigan (1993) for a detailed description of these fit- ting methods.

\section{EXAMPLES}

\subsection{Distribution Fitting without Data}

AbouRizk, Halpin and Wilson (1991) described an application of subjective distribution fitting in the context of construction simulation. To model the time required to resurface a section of runway at a small airport, the project engineer assembled the following information about this activity.

- Under the best circumstances, the minimum feasible time to complete the resurfacing is 1 hour.

- Resurfacing must be completed within an 8-hour period at night to avoid interfering with airport operations.

- In the past, similar resurfacing activities have most frequently lasted about 3 hours.

- There is at least a $75 \%$ chance that resurfacing will last at most 6 hours.

Using VIBES, the project engineer initially modeled the resurfacing time with a beta distribution having the characteristics specified above. Unfortunately, the resulting density appeared to be nearly uniform on the interval between 1 and 8 hours, and this model of the distribution of resurfacing times was judged to be unrealistic. Through a series of interactive modifications of the displayed beta density, the project engineer arrived at a more realistic model of the distribution of runway resurfacing time. Although the endpoints of the distribution were unchanged, the final fitted beta density had a much thinner right tail, with the 75 th percentile set at 4.61 hours.

We also used PRIME to model the distribution of the runway resurfacing time. First we defined the support of the distribution by changing the minimum and maximum values on the $x$-axis to 1 and 8 , respectively. Then we activated the PDF window and a statistics window showing the first four moments of the currently displayed distribution. We moved the control points from their default positions until the overall shape of the density was visually acceptable, and the mode was approximately 3 . Figure 4 shows the final fitted Bézier distribution with the CDF window on the left and the PDF window on the right. Table 1 displays the corresponding values for the mode, the 75 th percentile, the 99 th percentile, and the first four moments. In this example, the fitted Bézier distribution is very similar to the fitted beta distribution 


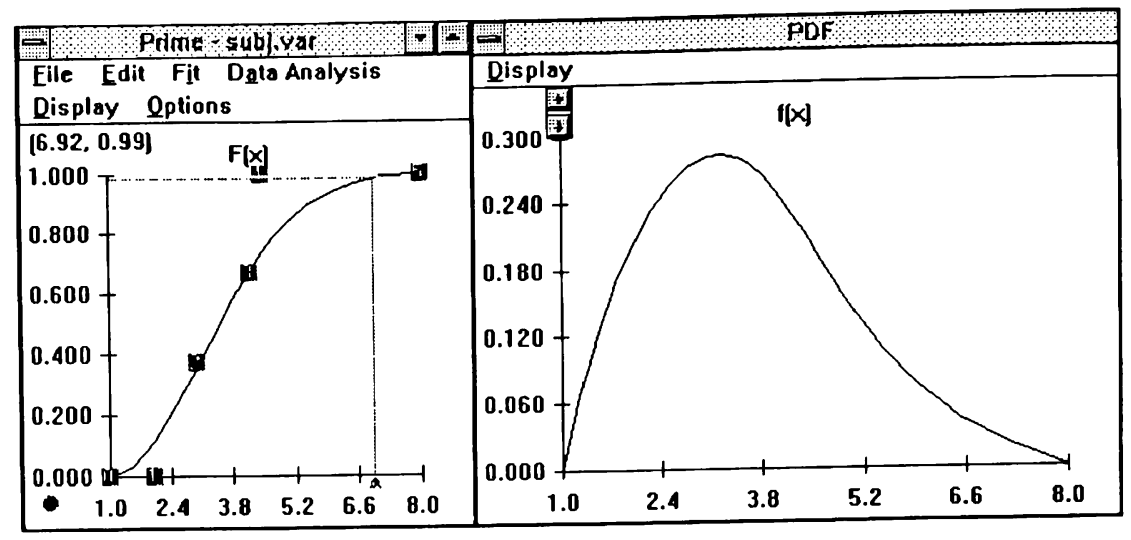

Figure 4: Subjectively Fitted Bézier Distribution of Runway Resurfacing Time

both visually and according to the characteristics displayed in Table 1.

Table 1: Comparison of Subjectively Fitted Bézier and Beta Distributions for Runway Resurfacing Time

\begin{tabular}{lcc}
\hline & Bézier & Beta \\
\hline Mean & 3.614 & 3.616 \\
Standard Deviation & 1.359 & 1.398 \\
Skewness & 0.482 & 0.368 \\
Kurtosis & 2.720 & 2.415 \\
Mode & 3.0 & 3.0 \\
99th percentile & 6.91 & 6.92 \\
75th percentile & 4.52 & 4.60 \\
Min, Max & 1,8 & 1,8 \\
\hline
\end{tabular}

\subsection{Distribution Fitting with Data}

As an example of distribution fitting with data, we describe an application of PRIME in a manufacturing simulation study. Surface mount capacitors are stored in lots of varying sizes in a facility adjacent to the insulation resistance (IR) testing area. To model the operation of the IR testing area, we needed to estimate the distribution of lot sizes in the storage facility.

Lot-size data were available for 2083 tested lots. Figure 5 displays a histogram and Figure 6 displays the empirical CDF for this data set, where all of the original observations were divided by 1000 for simplicity. Notice that in the vicinity of 20 and 270 on the new scale (that is, lot sizes expressed in 1000s), there are pronounced peaks in the histogram. Usually such a bimodal distribution indicates that the sample was taken from two distinct populations. In the current context, the production engineers were unable to provide any additional information that would enable us to model the lot-size distribution as a mixture of two simpler distributions; and thus we were forced to exploit the capabilities of PRIME for modeling multimodal distributions.

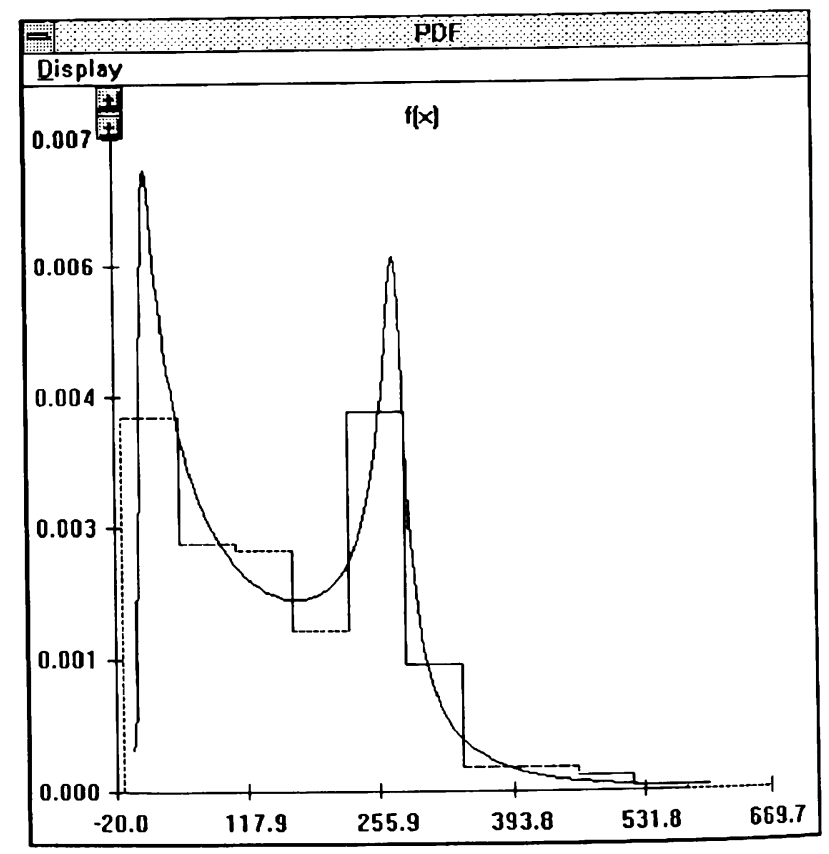

Figure 5: Least-Squares Fit for the Lot-Size Data Set

Once the lot-size data set was imported into PRIME, we moved the control points so that the currently displayed Bézier curve approximated the empirical CDF. Since the lot-size data set is bimodal, 


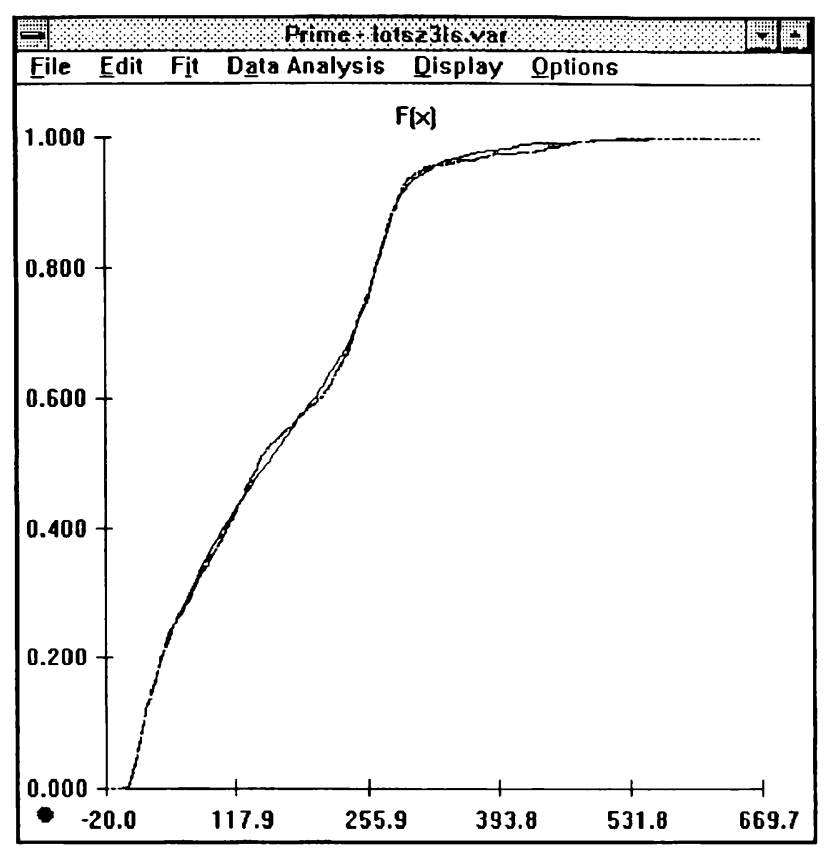

Figure 6: Least-Squares Fit for the Lot-Size Data Set

we judged that it would be easier to fit the sample data if the Bézier distribution had more than six control points (the default in PrIME); hence we created three additional control points. We then requested PRIME to find the least-squares fit to the empirical CDF. Figures 5 and 6 respectively display the resulting PDF and CDF from the Bézier distribution. The sum of the squared deviations of the fitted CDF from the empirical CDF is 0.0329, and the largest deviation between the fitted and empirical CDFs is 0.0237. Notice that the fitted PDF and CDF closely approximates the shape of the lot-size data set.

To demonstrate the advantages of using PRIME for fitting a distribution to the lot-size data set, we also obtained fits to this data set using FITTR1 and Unifit II. The FITTR1-generated PDF and CDF are displayed in Figures 7 and 8 respectively. Clearly the Johnson $S_{B}$ distribution obtained with FITTR1 is unacceptable-the single mode of the fitted distribution nearly coincides with the antimode of the empirical distribution that lies roughly halfway between the two modes mentioned earlier; and the maximum deviation between the fitted and empirical CDFs is 0.0862. Although the Johnson $S_{B}$ family includes some bimodal distributions, inherent restrictions on the relative placement of these modes effectively preclude the use of an $S_{B}$ distribution to represent the lot-size data set.

Unifit II selected the Weibull distribution as the

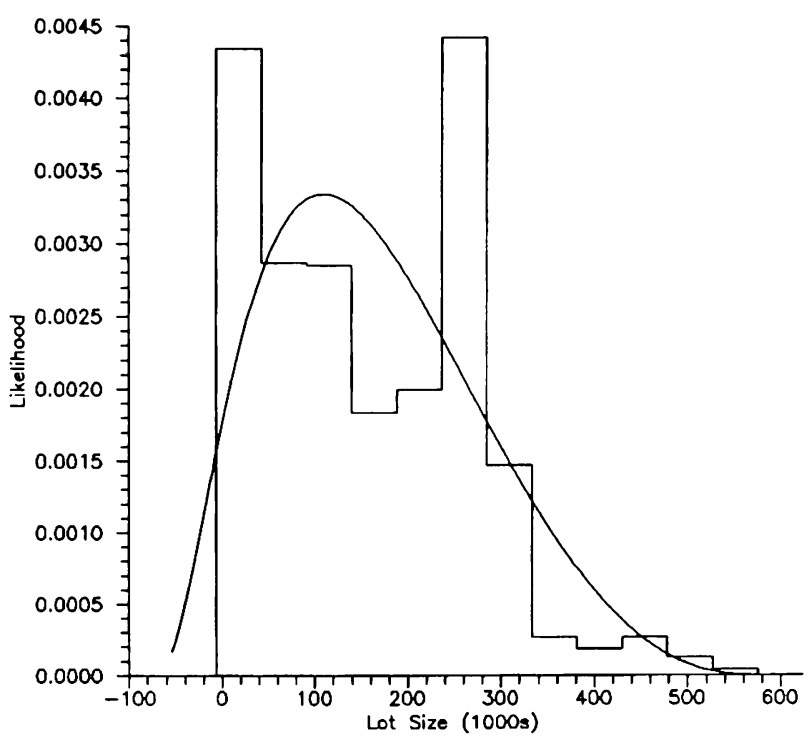

Figure 7: Minimum- $L_{\infty}$-Norm Fit to Lot-Size Data Set Using FITTR1 with an $S_{B}$ Distribution

best-fitting distribution for the lot-size data set. Figures 9 and 10 respectively display the resulting PDF and CDF. It is evident from these figures that the Weibull distribution selected by Unifit II is also unacceptable - the single mode of the fitted distribution nearly coincides with the first mode of the empirical distribution; and the second mode of the empirical distribution is largely ignored. The maximum deviation between the fitted and the empirical CDFs is 0.0695 ; and in fact, Unifit II does not recommend the Weibull distribution as a satisfactory representation of the data set.

\section{SUMMARY AND CONCLUSIONS}

Bézier distribution families are extremely flexible. If more degrees of freedom, or parameters, are desired, they may be easily incorporated into the formulation of the Bézier distribution. Increasing the number of parameters (control points) of a Bézier distribution simply provides more flexibility without changing the form of the distribution, which is still Bézier.

Distribution fitting using Bézier functions combines all available information-subjective, visual, and empirical-in the formulation of the distribution. If data are available, then automatic distribution fitting via conventional methods (such as least-squares, moment-matching, and maximum-likelihood estimation) is readily performed. 


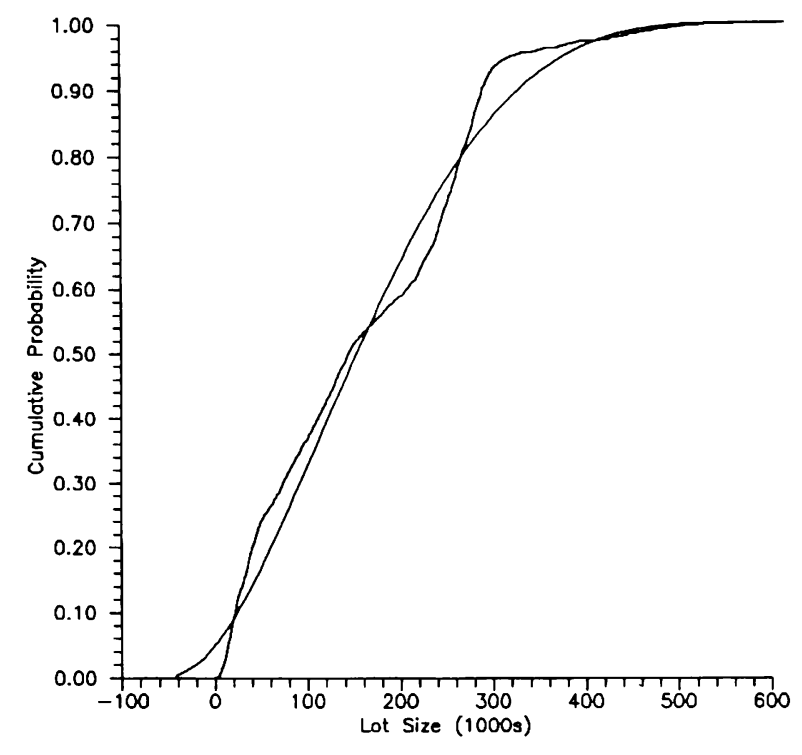

Figure 8: Minimum- $L_{\infty}$-Norm Fit to the Lot-Size Data Set Using FITTR1 with an $S_{B}$ Distribution

\subsection{Univariate Distribution Families}

If $X$ is a continuous random variable having a univariate Bézier distribution function $F_{X}(\cdot)$ as defined in $\S 3.2$, then the distribution of $X$ has the following properties:

- The first control point, $\boldsymbol{p}_{0} \equiv\left[x_{0}, z_{0}\right]$, and the last control point, $\boldsymbol{p}_{n} \equiv\left[x_{n}, z_{n}\right]$, are exactly interpolated. This property ensures that $F_{X}\left(x_{0}\right)=z_{0}$ and $F_{X}\left(x_{n}\right)=z_{n}$; and when $z_{0} \equiv 0$ and $z_{n} \equiv$ 1.0 , a valid CDF can be constructed.

- The density function, $f_{X}(\cdot)$, has a closed-form parametric representation as a ratio of two Bézier curves, as given by (3).

- The density function is determined from the set of control points, $\left\{\boldsymbol{p}_{0}, \boldsymbol{p}_{1}, \ldots, \boldsymbol{p}_{n}\right\}$, that define the CDF. The result is an exact parametric representation of the density-no information is lost or approximated in the conversion.

- The $k$ th noncentral moment, $E\left[X^{k}\right]$, of a Bézier random variate $X$ has a closed-form solution, and it also has a computationally efficient expression given by (7).

\subsection{Modeling Simulation Inputs with PRIME}

PRIME implements the methodology discussed in $\$ 3$ so that users may construct univariate distributions based on a family of curves in Bézier form. This

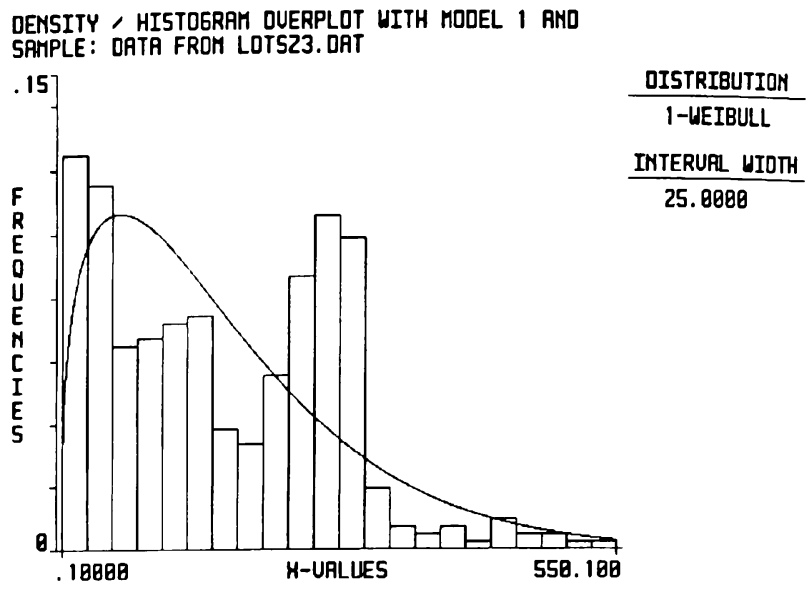

Figure 9: Unifit II Fit to the Lot-Size Data Set with a Weibull Distribution

\section{OISTRIBUTION FUNCTION PLOT OF MODEL 1 AND} SAMPLE: DATA FROM LOTSZ3. DAT

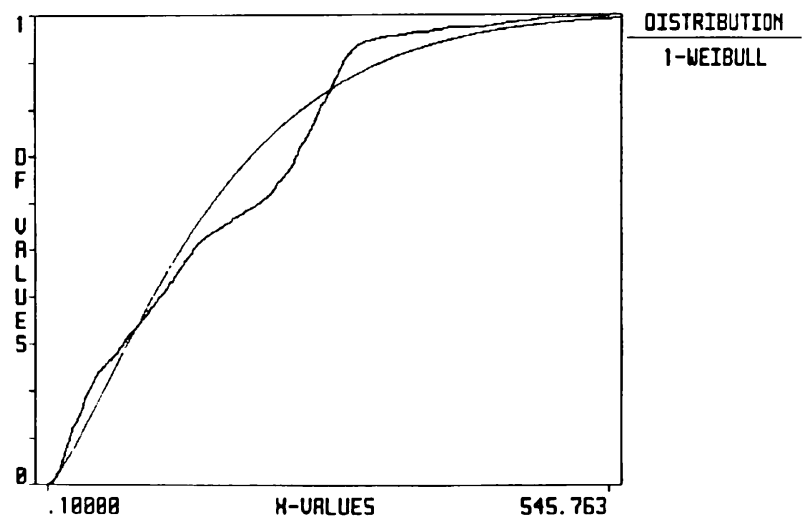

Figure 10: Unifit II Fit to the Lot-Size Data Set with a Weibull Distribution

methodology is implemented in a graphical environment.

From the user's point of view, PrIME is an easyto-use, intuitive, graphical software system. PrImE presents immediate, visual feedback on the currently configured distribution. The user can easily alter an inappropriately-configured distribution by adding, deleting, or relocating one or more of the control points given in (1).

\section{ACKNOWLEDGMENTS}

This work was partially supported by a David Ross Grant from the Purdue Research Foundation and by NSF Grant No. DMS-8717799. The authors thank 
Steve Roberts, Bruce Schmeiser, and Arnold Sweet for many enlightening discussions on this paper.

\section{REFERENCES}

AbouRizk, S. M., D. W. Halpin, and J. R. Wilson. 1991. Visual interactive fitting of beta distributions. Journal of Construction Engineering and Management 117 (4): 589-605.

Avramidis, A., and J. R. Wilson. 1989. A flexible method for estimating inverse distributions in simulation experiments. In Proceedings of the 1989 Winter Simulation Conference, ed. E. A. MacNair, K. J. Musselman, and P. Heidelberger, 428-436. Institute of Electrical and Electronics Engineers, Piscataway, New Jersey.

Avramidis, A., and J. R. Wilson. 1993. A flexible method for estimating inverse distributions in simulation experiments. ORSA Journal on Computing to appear.

Bratley, P., B. L. Fox, and L. E. Schrage. 1987. A guide to simulation. 2d ed. New York: SpringerVerlag.

Conte, S. D. and C. de Boor. 1980. Elementary numerical analysis: An algorithmic approach. $3 \mathrm{~d}$ ed. New York: McGraw-Hill.

Conway, R., W. L. Maxwell, J. O. McClain, and S. L. Worona. 1987. User's guide to XCELL+factory modeling system. $2 \mathrm{~d}$ ed. Redwood City, California: The Scientific Press.

DeBrota, D. J., R. S. Dittus, S. D. Roberts, and J. R. Wilson. 1989. Visual interactive fitting of bounded Johnson distributions. Simulation 52 (5): 199-205.

Farin, G. 1990. Curves and surfaces for computer aided geometric design: A practical guide. $2 \mathrm{~d}$ ed. New York: Academic Press.

Flanigan, M. A. 1993. A flexible, interactive, graphical approach to modeling stochastic input processes. Ph.D. diss., School of Industrial Engineering, Purdue University, West Lafayette, Indiana.

Hora, S. C. 1983. Estimation of the inverse function for random variate generation. Communications of the ACM 26 (8): 590-594.

International Mathematical and Statistical Library. 1987. IMSL library reference manual. 10th ed. Houston: IMSL, Inc.

Law, A. M., and W. D. Kelton. 1991. Simulation modeling and analysis. 2d ed. New York: McGrawHill.

Microsoft Corporation. 1990. User's manual for the software development kit. Redmond, Washington: Microsoft Corporation.

Roberts, S. D. 1983. Simulation modeling and analysis with INSIGHT. Indianapolis: Regenstrief Insti- tute for Health Care.

Swain, J. J., S. Venkatraman, and J. R. Wilson. 1988. Least-squares estimation of distribution functions in Johnson's translation system. Journal of Statistical Computation and Simulation 29:271-297.

Vincent, S. G., and W. D. Kelton. 1992. Distribution selection and validation. In Proceedings of the 1992 Winter Simulation Conference, ed. J. J. Swain, D. Goldsman, R. C. Crain, and J. R. Wilson, 300-304. Institute of Electrical and Electronics Engineers, Piscataway, New Jersey.

Vincent, S. G., and A. M. Law. 1992. UniFit II: Total support for simulation input modeling. In Proceedings of the 1990 Winter Simulation Conference, ed. J. J. Swain, D. Goldsman, R. C. Crain, and J. R. Wilson, 371-376. Institute of Electrical and Electronics Engineers, Piscataway, New Jersey.

\section{AUTHOR BIOGRAPHIES}

MARY ANN FLANIGAN WAGNER is currently working at SysTech Software Solutions, located in Annandale, Virginia. Her responsibilities include the continuing development and marketing of the simulation language INSIGHT. Her principal interests are in simulation development, modeling and analysis. Her undergraduate and graduate degrees are in the field of Industrial Engineering, and in May 1993, she received her Ph.D. from Purdue University. From 1987 to 1989 she held a research position at the Regenstrief Institute, where she was responsible for the development and analysis of simulation models. Dr. Wagner is a member of: Alpha Pi Mu, ACM, IIE, Omega Rho, ORSA, SIGGRAPH, SIGSIM, and TIMS.

JAMES R. WILSON is a Professor in the Department of Industrial Engineering at North Carolina State University. He received a B.A. degree in mathematics from Rice University, and he received M.S. and Ph.D. degrees in industrial engineering from Purdue University. His current research interests are focused on the design and analysis of simulation experiments. He also has an active interest in applications of operations research techniques to all areas of industrial engineering. From 1988 to 1992 , he served as Departmental Editor of Management Science for Simulation. He was Proceedings Editor for WSC '86, Associate Program Chair for WSC '91, and Program Chair for WSC '92. He has also held various offices in TIMS/College on Simulation. He is a member of ASA, ACM/SIGSIM, IIE, ORSA, SCS, and TIMS. 